Rev Inv Vet Perú 2000; 11(2):44-50

\title{
FACTORES DE RIESGO EN LA PRESENTACIÓN DE HEMORRAGIA PULMONAR INDUCIDA POR ESFUERZO (EIPH) EN CABALLOS DE CARRERA
}

\author{
Eduardo Jiménez Z.' y Sergio Cueva M. ${ }^{2}$
}

\section{Abstract}

The potential risk factors associated with development of Exercise-Induced Pulmonary Hemorrhage (EIPH) in Thoroughbred horses are evaluated. This process affects a horses performance and therefore its capacity for competition. Regression analysis of data collected between 1996 and 1998 for 4000 horses, (age, sex, distances run, season of the year and surface of the track, and health status), including 49 animals which exhibited clinical EIPH, revealed that mares are particularly prone to the disease. Key Words: Exercise-Induced Pulmonary Hemorrhage (EIPH), thoroughbred horse.

\section{Resumen}

El presente trabajo tuvo por finalidad analizar algunos factores que pudieran representar un riesgo potencial para que los equinos de carrera desarrollen un proceso patológico denominado Hemorragia Pulmonar Inducida por Esfuerzo (EIPH). Dicho proceso afecta de manera fehaciente el rendimiento del ejemplar, disminuyendo su capacidad de competencia. El estudio es de carácter retrospectivo y acumula datos de los años 1996, 1997 y 1998, referentes a la edad y el sexo del ejemplar, así como la distancia, época del año y tipo de pista sobre la que se desarrollaron dichas competencias. Asimismo, se consigna si el ejemplar sufrió de EIPH. De los 4000 ejemplares evaluados, 49 mostraron signos clínicos de la enfermedad. Los datos recabados fueron sometidos a un análisis estadístico de Regresión Logística a fin de determinar los factores que influyeron sobre la presentación del problema. Como resultado del trabajo se determinó que el sexo, específicamente el ser hembra, es un factor de riesgo para la presentación de EIPH.

Palabras Clave: Hemorragia Pulmonar Inducida por Esfuerzo (EIPH), caballo de carrera.

La Hemorragia Pulmonar Inducida por Esfuerzo (EIPH) es un serio problema en la industria del caballo Pura Sangre de Carrera (PSC). Asimismo, afecta otras razas como los Standardbreds (25-40\%), los Pony Club Horses (más del 10\%), los caballos del evento de los 3 días (40\%), Steeplechasers (más del 70\%), Polo Ponies

\footnotetext{
'Práctica privada

'Laboratorio de Fisiología-FMV - UNMSM
}

(10-15\%), y los Quarter Horses (65\%) (Hinchcliff, 1998; Sellnow, 1997). Desde el tiempo de la Reina Elizabeth de Inglaterra (s. XVIII) habían caballos que sangraban por la nariz (epistaxis) durante el galope. Durante más de 100 años se creyó que éste síndrome estaba asociado a hemorragias de la cavidad nasal causadas por fragilidad capilar o coagulopatías. Una de las razones para la persistencia de esta concepción equivocada se debe al hecho de que las hemorragias de origen pulmonar en el caballo, 
debido a la disposición más bien horizontal del árbol respiratorio, fluyen libremente hacia la nariz sin formar espuma ni causar accesos de tos, como ocurre en humanos (Andresen, 1992).

La EIPH es el inevitable resultado del conflicto de los requerimientos de una barrera alvéolo-capilar extremadamente delgada y la elevada presión de llenado del ventrículo izquierdo, la cual aparentemente es necesaria para las extraordinarias rendimientos aeróbicos de éstos atletas.

West et al. (1997) señalan que la epistaxis en PSC es una condición de hiperemia de los capilares pulmonares, algunas veces acompañada de extravasación desde éstos a los sacos aéreos y tejido conectivo. Un siglo después, las observaciones hechas mediante microscopía electrónica por West et al. (1992) han provisto evidencia de que la ruptura de los vasos pulmonares ocurre y hay al menos algún grado de edema intersticial (West et al., 1997).

En 1913, en un análisis del posible papel hereditario de la epistaxis en PSC, se postuló que si la lesión ocurre en la parte superior de la cavidad nasal, algunas veces algo de la sangre es llevada por el aire inspirado a la glotis, y en algunos casos llega a los pulmones (West et al., 1997).

En 1974, Cook comprobó evidencia de que la fuente de hemorragia en caballos "sangradores" era el pulmón; al respecto hay dos posibilidades: o el ejercicio causa ruptura de los vasos sanguíneos o hay desplazamiento de los eritrocitos desde los vasos sanguíneos intactos (diapedesis) (West et al., 1997).

No fue hasta la década del ' 70 que se usó el broncoscopio flexible y se supo que el sangrado de los pulmones era muy común (alrededor del $44 \%$ de los caballos que galopaban) (West et al, 1997). Luego, se mostró la existencia de macrófagos con hemosiderina en lavados traqueales, lo que demostraba la presencia de sangre en los pulmones. De esta manera, los caballos que sangran profusamente disminuyen su rendimiento atlético, por lo cual la EIPH es uno de los más graves problemas en esta industria (West $e t$ $a l, 1997)$.

La epistaxis por lo general es bilateral e igualmente distribuida por ambos ollares. Sin embargo, en la gran mayoría de los casos la hemorragia pulmonar es subclínica, permaneciendo la sangre secuestrada en los pulmones (West et al., 1997). Aproximadamente, 1 de cada 9 caballos con EIPH muestra sangre por los ollares después de la carrera (Hinchcliff, 1998). En algunos casos la sangre no pasa a la nariz y es deglutida, lo que puede comprobarse por la presencia de sangre oculta en las heces (prueba de la bencidina) (Andresen, 1992).

Entre las causas de EIPH se consideran: infestación parasitaria, enfermedad pulmonar crónica, patógenos sanguíneos, obstrucción parcial de las vias aéreas superiores, defectos de la coagulación, asfixia, y mecanismos de estrés en el parénquima pulmonar durante el ejercicio fuerte (Cook et al., 1997; Jones, 1998). Asi mísmo, se cree que hay un componente genético de por medio (inbreeding) (Andresen, 1992). El abuelo del gran Jefe de Raza Eclipse sufría de constantes hemorragias, por lo que le cambiaron el nombre de Bleeding (sangrador) Childers a Bartlett's Childers a fin de que fuera aceptado en los registros como uno de los fundadores de la raza. De esta manera, el componente genético de la EIPH fue transmitido a lo largo de sucesivas generaciones (Veterinary Supplement, 1998). También se reporta que el gran Jefe de la Raza Hermit (s. XIX) manifestaba la tendencia a sufrir de hemorragias (Zamalloa, 1979). Posteriormente West et al. (1997) reportaron en pulmones de 26 pura sangre de carrera (PSC) con EIPH la presencia de lesiones multifocales con hemosiderófagos y aumento de tejido conectivo. Ellos sugieren que la etiología fue un ligero grado de bronquiolitis, posiblemente de origen viral, y sostienen que el sangrado 
proviene de la neovascularización de la circulación bronquial (West et al., 1997).

En cuanto a la influencia de la edad Zamalloa (1979), sostiene que la EIPH es más frecuente en animales jóvenes, y que el problema desaparece con la edad, probablemente con el paulatino desarrollo y adaptación de los sistemas locomotor, cardiovascular y endocrino del animal. Sin embargo, reportes posteriores sostienen que el problema afecta de igual modo en todas las edades.

Con respecto al sexo, West et al. (1997), hicieron un estudio en el que encontraron 59 yeguas, 40 capones y 25 machos que sufrieron de EIPH, principalmente en primavera y otoño, concluyendo que la mayor frecuencia en las hembras se debe a que éstas sufren variaciones periódicas en sus niveles de estrógenos y progesterona (Zamalloa, 1979). Empero, en humanos, casi todos los valores cuantitativos para las mujeres (fuerza muscular, ventilación pulmonar, gasto cardiaco) relacionados principalmente con la masa muscular, varían entre $2 / 3$ y $3 / 4$ de los valores registrados para los varones (Guyton, 1992). Por lo que es de esperar que la presión arterial media durante el ejercicio sea también menor en las hembras que en los machos, como lo indican los rendimientos comparativos en similares eventos entre ambos sexos.

Goetz et al. (1996), analizaron un grupo de 15 caballos mediante examen endoscópico semanal, 55 ó 60 minutos luego de ser sometidos a ejercicio a una velocidad de $14.5 \mathrm{~m} / \mathrm{s}$. a fin de diagnosticar EIPH; asimismo, controlaron el ritmo cardiaco y las presiones del atrio derecho y la arteria pulmonar, reportando 7 animales que desarrollaron EIPH después del ejercicio y 8 que nunca dieron síntomas del problema; sin embargo, no hubo diferencias estadísticas entre los valores obtenidos para el ritmo cardiaco y las presiones registradas entre los animales con EIPH y aquellos que no la tuvieron.
En un estudio realizado en capones, se sometió a éstos a ejercicios durante 15 días a velocidades de $9,11,13$ y $15 \mathrm{~m} / \mathrm{s}$. Luego de una hora de ejercicio, se hicieron lavados broncoalveolares y se cuantificó el número de células rojas en el fluído. No hubo variación significativa entre los animales sometidos a las 3 primeras velocidades, pero en aquellos a los que se ejercitó a $15 \mathrm{~m} / \mathrm{s}$ se observó un significativo aumento en el número de eritrocitos presentes en el lavado (Langsetmo, 1996).

En el estudio de West et al. (1997) se señala, la fibrosis y depósitos de hemosiderina asociados con viejas lesiones de EIPH son principalmente encontrados en la región dorso-caudal del pulmón (Equine Reports, 1998). A priori, se esperaría que las presiones capilares fueran menores en la región dorsal del pulmón con respecto a la ventral, debido al gradiente de presión hidrostática. No obstante, se presume que hay otros factores importantes.

Acerca de este punto, hay una teoría que propone que el impacto de los cascos en el terreno es transmitido por los miembros a la pared torácica, desde donde una compleja serie de ondas son generadas en el pulmón. Estas ondas son transmitidas desde la parte frontal del pulmón a la caudal, siendo reflejadas por las costillas y la espina dorsal y amplificadas en varias partes del pulmón, causando fractura de capilares y el sangrado (Schroter y Marlin, 1998). Es posible que en el caballo la vasculatura pulmonar en la región dorso-caudal sea diferente a la de cualquier otra porción del órgano. Algunos estudios muestran que el flujo sanguíneo pulmonar es especialmente alto en esta región cuando el animal está en posición normal (prona). Asimismo. podría ser que ésta región tenga una resistencia vascular intrínseca menor. Estudios hechos de falla por estrés en pulmones aislados de PSC mostraron que si la presión transmural capilar excede los 50 $\mathrm{mmHg}$ el número de rupturas en el epitelio alveolar tiende a ser sistemáticamente mayor en las porciones dorsales que en las ventrales (Pelletiner, 1997). 
Otro mecanismo para la localización dorso-caudal de la EIPH es que los alvéolos dorsales son más largos que los de cualquier otra región en el pulmón por la distorsión que ocurre en el pulmón como resultado de su peso. Se ha observado que la mayor presión negativa pleural en el caballo ocurre en la región dorso-caudal (West et al., 1997).

Aparentemente, en los PSC la falla ocurre en todos los animales en entrenamiento; claramente la falla podría ser catastrófica para el intercambio gaseoso si los alvéolos se llenaran con sangre. La barrera alvéolo-capilar ofrece 2 mecanismos contrarios: es adecuadamente delgada para permitir un eficiente intercambio gaseoso por difusión y, a la vez, no es lo suficientemente delgada (o no tiene una superficie lo suficientemente extensa) para brindar rangos óptimos de difusión de oxígeno durante el ejercicio extremo, ya que todos los PSC desarrollan hipoxemia arterial severa durante el galope como resultado de la difusión limitada a través de la barrera. Por ésto, hay una clara ventaja para la performance aeróbica en tener una barrera alvéolo-capilar delgada y presumiblemente, si se seleccionan animales basándose en su performance superior se favorecería a aquellos con barrera alvéolo-capilar delgada (West et al., 1997).

Sin embargo, la barrera alvéolo-capilar debe ser lo suficientemente resistente para reducir la probabilidad de falla mecánica. No obstante, todos los PSC desarrollan alguna falla por estrés en sus capilares pulmonares durante el entrenamiento, como se evidencia por los macrófagos con hemosiderina presentes en lavados traqueales (West et al., 1997).

El objetivo del presente estudio fue realizar un análisis retrospectivo de la información de competencias oficiales de equinos de carrera a fin de determinar si el sexo y la edad, así como la época del año, distancia y tipo de pista en que se desarrolló la competencia constituyeron factores de riesgo para la presentación de casos de EIPH, usando como método de diagnóstico la observación clínica de epistaxis luego de concluido el evento.

\section{Materwes t Wrowns}

En el presente estudio se recolectaron datos de 4000 equinos de carrera que actuaron en competencias oficiales en el Hipódromo de Monterrico (Surco-Lima), durante los años 1996, 1997 y 1998. Para este fin se consultaron los Calendarios Oficiales de las carreras disputadas en estos años, y además se constató el Parte Veterinario Oficial semanal, durante el periodo que abarcó el estudio para determinar los ejemplares que habían sufrido de EIPH. Dicho diagnóstico se había llevado a cabo mediante la detección de la presencia de epistaxis en los ejemplares luego de concluida la competencia.

Para la selección de los animales incluidos en las muestras, se consideró el número total de competidores que corrieron, durante los 3 años, por trimestres (Cuadro 1).

Cuadro 1. Número de caballos que corrieron, por trimestre, durante los tres años.

\begin{tabular}{ccc}
\hline Año & Trimestre & $\begin{array}{c}\text { Competidores, } \\
\mathrm{n}\end{array}$ \\
\hline \multirow{2}{*}{1996} & $1^{\circ}$ & 5066 \\
& $2^{\circ}$ & 5067 \\
& $3^{\circ}$ & 5033 \\
1997 & $4^{\circ}$ & 5171 \\
& $1^{\circ}$ & 4976 \\
& $2^{\circ}$ & 4642 \\
& $3^{\circ}$ & 4660 \\
1998 & $4^{\circ}$ & 4717 \\
& $1^{\circ}$ & 4627 \\
& $2^{\circ}$ & 4608 \\
& $3^{\circ}$ & 4310 \\
& $4^{\circ}$ & 4336 \\
\hline \multirow{2}{*}{ Total } & & 57213 \\
\hline
\end{tabular}

Con estos valores se procedió a calcular el número de competidores a considerarse 
en la muestra de cada trimestre, usando la siguiente fórmula:

$$
(n / N) \times 4000=X
$$

donde $\mathbf{n}$ es el número de competidores que corrieron en determinado trimeste, $\mathrm{N}$ el número total de participantes que compitieron en los tres años de estudio, $4000 \mathrm{el}$ número de competidores considerados en la muestra, y X el número de competidores seleccionados en cada trimestre.

Así, la muestra total quedó constituída por el número de competidores seleccionados en cada trimestre, según se aprecia en el Cuadro 2.

Cuadro 2. Muestra total de competidores seleccionados en cada trimestre por 3 años

\begin{tabular}{ccc}
\hline Año & Trimestre & $\begin{array}{c}\text { Competidores, } \\
\mathrm{n}\end{array}$ \\
\hline \multirow{2}{*}{1996} & $1^{\circ}$ & 354 \\
& $2^{\circ}$ & 354 \\
& $3^{\circ}$ & 352 \\
1997 & $4^{\circ}$ & 362 \\
& $1^{\circ}$ & 348 \\
& $2^{\circ}$ & 324 \\
& $3^{\circ}$ & 326 \\
1998 & $4^{\circ}$ & 330 \\
& $1^{\circ}$ & 324 \\
& $2^{\circ}$ & 322 \\
& $3^{\circ}$ & 302 \\
& $4^{\circ}$ & 302 \\
\hline Total & & 4000 \\
\hline
\end{tabular}

Finalmente, con el objetivo de realizar un muestreo sistemático, se calculó el intervalo entre un competidor seleccionado de la muestra y el siguiente dividiendo el número total de participantes que compitieron en cada trimestre entre el número de animales que debían ser seleccionados para la muestra de cada trimestre. En todos los casos dicho valor fue 14. Los datos colectados fueron la edad, el sexo, la época (trimestre) del año en que se efectuó la competencia, así como la pista (arena o césped) y la distancia sobre la que se llevó a cabo. Con respecto a la distancia, se agruparon en tres categorías de acuerdo al promedio de velocidades que se registran en cada una de ellas. Se tomó como referencia la Tabla de récord del Hipódromo de Monterrico.

Una vez tabulados todos los datos, fueron sometidos al análisis de Regresión Logísti$\mathrm{ca}$, usando el programa SPSS versión 7.5, a fin de determinar si la presentación de EIPH guardaba relación con alguno(s) de los factores de riesgo propuestos. En este caso, la variable dependiente fue EIPH (presencia o no de la enfermedad).

\section{Resultados y Discusión}

De acuerdo al análisis realizado, el único factor que influye en la presentación de EIPH es el sexo (Cuadro 3). Más especificamente, el ser hembra predispone al ejemplar 1.82 más veces que ser macho a sufrir de la enfermedad. Como explicación se piensa que la causa es la fluctuación periódica en los niveles de estrógenos y progesterona en las hembras (Jubiz, 1996). Los estrógenos y sus derivados pueden elevar la presión arterial en algunos casos (Rosenstein, 1999). Aunque no está claro éste mecanismo, se cree que los estrógenos estimulan la formación de sustrato de renina y la activación del sistema renina-angiotensina-aldosterona, aumentando la reabsorción de sodio a nivel de los túbulos renales, con la consiguiente retención de líquido y elevación de la presión arterial (García, 1996).

Cuadro 3. Distribución de la muestra por sexos.

\begin{tabular}{crc}
\hline EIPH & Machos & Hembras \\
\hline Con & 20 & 29 \\
Sin & 2199 & 1752 \\
\hline Total & 2219 & 1781 \\
\hline
\end{tabular}


Los factores de edad, pista, distancia y época del año no han podido ser apropiadamente evaluados debido a que el número de casos de HIPH obtenidos del total de la muestra (49 de 4000$)$ no es suficiente para precisar el rol que juegan. Esto es a pesar de que la proporción de casos de EIPH registrados en la muestra con respecto al tamaño total de ésta fue de $1.23 \%(49 / 4000)$ y guarda relación con el número de casos total $(1.20 \%$ 686/57 213) que se registró durante los 3 años de estudio. Estas cifras se corresponden en cierto grado con la baja incidencia de $6.1 \%$ encontrada usando el mismo método de diagnóstico en nuestro medio (Zamalloa, 1979).

Debemos precisar que el diagnóstico de EIPH se hizo mediante la observación visual después de la competencia, dejando de lado aquellos animales sospechosos de haber sufrido hemorragia o aquellos que la experimentaron pero que no manifestaron la epistaxis. A este respecto, hay un estimado que formula que solamente 1 de cada 9 caballos que sufren de EIPH muestran el sangrado por los ollares (Hinchcliff, 1998). A pesar de ésto, solamente el $10.8 \%$ del total de ejemplares que compitieron sufririan presumiblemente de EIPH, contrastando con los valores encontrados de 55\% (Anónimo, 1999) y $75-90 \%$ (Andresen, 1992), respectivamente.

Asimismo, hay que destacar que salvo el trabajo de Zamalloa (1979), los trabajos hechos sobre éste proceso son de tipo prospectivo, lo cual dificulta enormemente la posibilidad de conseguir un número de casos suficiente para someterlo a un análisis estadístico conveniente. Sin embargo, la mejor manera de estudiar la EIPH es haciendo el diagnóstico mediante la bronquioscopía, lo cual se realizaría de manera prospectiva, pues no es práctica habitual en el hipódromo realizar ésta después de las competencias salvo contados casos, debido sobretodo al costo que esta representaría para cada propietario de caballos.

\section{Conclusiones}

La EIPH es un proceso patológico influenciado por el sexo, el ser hembra le da al ejemplar 1.82 veces más probabilidades de sufrir de EIPH que si fuera macho.

Muchos casos subclínicos se pierden al momento del muestreo debido a que no se realizan regularmente controles de los ejemplares mediante broncoscopia una vez finalizada la competencia. Dicho examen debe ser hecho dentro de las 24 horas posteriores a la competencia.

Debido a la conclusión anterior, la HIPH debe ser estudiada de manera prospectiva, a fin de que la frecuencia de presentación del problema no se diluya dentro del total de la muestra.

\section{Literatura Citada}

1. Andresen, H. 1992. Manual de las enfermedades de los equinos. $1^{\text {a }}$. Ed., p. 8082. La Moderna-Perú.

2. Anónimo. (1999). Excercised-Induced Pulmonary Haemorrhage. (Online). Disponible: http://www.aht.org.uk/ R\%20respiratory\%20studies.htm

3. Anónimo. 1998. Respiratory System. Veterinary Supplement . (Online), 27 par. Disponible: http://www. equineonline.com/misc/ glosvetsup.html

4. Cook, W. et al. 1997. Etiology of EIPH in the horse. En Grayson Equine Medical Research Database. (Online) Disponible: http://www.jockeyclub. com/gravsondb/card.html (12/09/1999)

5. García, A. 1996. Fisiología Veterinaria. $1^{\mathrm{a}}$. Ed., p. 851. Mc Graw-Hill - España.

6. Goetz, M. et al. 1996. Similarity of pulmonary vascular pressures of exercising thoroughbred horses with and without endoscopic evidence of EIPH. Journal of Applied Physiology. (Online), 19(7), 8 par. Disponible http:/l www.uth.tmc.edu/apstracts/1996/jap/ 
July/315.html (13/09/1999)

7. Guyton, $A$. 1992. Tratado de fisiología médica $2^{a}$. Ed., p. 983. Interamericana-España.

8. Hinchcliff, $K, \quad$ 1998. Exercise Induced Pulmonary Haemorrhage (EIPH): Is it a Disease? The Horse. (Online), 15 par. Disponible: httn://www.thehorse.com/ convention reports/weas $98 /$ einh.html

9. Jones, $W$. 1998. Air Flow: An Important Limitation for Racehorses. The Horse. (Online), 18 par. Disponible: http: /www.thehorse.com/airflow0898.htm

10. Jubiz, W. 1996. Endocrinología clínica. $3^{\text {a }}$. Ed., p. 532-533. El Manual ModernoMéxico.

11. Langsetmo, I. (1996). Relationship between pulmonary vascular pressure and pulmonary haemorrhage in the horse: Is there a threshold value? Journal of Applied Physiology. (Online), 19(5), 5 par. Disponible:http://www.uth.tmc. edu/apstracts/1998/iap/February/ 533A.html

12. Pelletiner, N. (1997). Regional differences in Equine Pulmonary Arterial End othelium-mediated responses: possible role in the distribution of EIPH.
En Grayson Equine Medical Research Database. (Online). Disponible: http:// www.jockevclub.com/gravsondb/ card.html

13. Rosenstein, E. 1999. Diccionario de Especialidades Farmacéuticas. 11 ${ }^{\mathrm{a}}$. Ed., p. 463. PLM-Perú.

14. Schroeter, B., Marlin, D. 1998. New theory explains why horses bleed during exercise. Technology and Medicine. (Online), par. Disponible:http:/l www.ad.ic.ac.uk/xr/media/ pressrel/ archive/eiph.html

15. Sellnow, L. 1997. Exercise-Induced Pulmonary Haemorrhage. The Horse. (Online), 29 par. Disponible: http:// www.thehorse.com/current/ eiph0997.html

16. West, J. et al. 1997. Excersice-Induced Pulmonary Haemorrhage. Proceedings of the second Dubai International Equine Symposium: The Diagnosis and Treatment of Respiratory Disease. $p$. 356-366.

17. Zamalloa, L. 1979. Contribución al estudio de la Epistaxis en el Caballo Pura Sangre de Carrera en nuestro medio. Tesis Bachillerato. Fac. Med. Vet. Univ. Nac. Mayor San Marcos, Lima. 52 p. 